\title{
REPORT ON OVERLAP METHODS IN PHOTOGRAPHIC ASTROMETRY
}

\author{
Chr. DE VEGT
}

The usual procedure for deriving standard coordinates $\xi, \eta$ from the measured rectangular coordinates $x, y$ of a star is to adopt a suitable model for the relationship between the measured $x, y$, magnitude $m$, color index $c$ and the $\xi, \eta$. This relationship may, in general, be written as

$$
\xi=\sum a_{i j k l} x^{i} y^{j} m^{k} c^{l} ; \quad \eta=\sum b_{i j k l} x^{i} y^{j} m^{k} c^{l} .
$$

From the known standard coordinates $\xi_{\mathrm{r}}, \eta_{\mathrm{r}}$ of some reference stars equally distributed over the plate area, the unknown plate constants $a_{i j k l}, b_{i j k l}$ are found from a least-squares adjustment. After the adjustment the standard coordinates of each field star can be computed. In most cases a statistical criterion for the choice of the proper form of the above relationship between measured and standard coordinates may be useful because the systematic accuracy, obtained from the adjustment, may be seriously affected by the number of unknown parameters included. (Eichhorn et al., 1967). If the same star occurs on two or more plates, i.e. the plates being partly overlapped, one gets the final position by taking the mean of the individual positions on each plate. In this procedure, no direct account is taken of the 'overlap' in the equations of condition for the desired plate constants.

It was first proposed by Eichhorn (1960) to include the positions of the field stars according to the condition that $\xi_{i}^{1}=\xi_{i}^{2}$ and $\eta_{i}^{1}=\eta_{i}^{2}$ for each star appearing in the overlap region of the plate 1 and 2 , and referring all plates to a common tangential point - in the equations of condition, and solve for the field stars and plate constants together as unknowns.

Recently, Googe (1967) has modified this procedure by an elimination of the field stars from the normal equations so that the overlap condition is expressed by the plate constants themselves. Practical results of Eichhorn's method are now available (cf. Eichhorn and Gatewood, 1967) from a new reduction of the Northern Hyderabadzone of the Astrographic Catalogue. A part of the material (103 plates) was subjected to an adjustment according to the plate-overlap method in groups of 6 plates each. It could be shown that, in comparison with the classical reduction of the individual plates, the overlap reduction is most effective in increasing the weight of coma and rotation coefficients, whereas zero point and magnitude effects are affected least. As an important result, it is found that an overlap solution using about $\frac{1}{3}$ of the reference

Perek (ed.), Highlights of Astronomy, 343-346. (c) I.A.U. 
stars yields positions with the same systematic accuracy as a classical solution involving the full number of reference stars.

A somewhat different treatment of the problem has been given by Lacroute (1964). Assuming that an initial set of plate constants has been found, the differences $\Delta \alpha=$ $\alpha_{v}^{i}-\alpha_{v}^{j}, \Delta \delta=\delta_{v}^{i}-\delta_{v}^{j}$ ( $v$ runs over all common stars of the plate-pair $(i, j)$ ) from the resulting individual positions on the overlapping plates are used to set up equations of condition for differential corrections of the plate constants. The possibility of directly handling the spherical coordinates themselves instead of using standard coordinates, is due to a first order approximation of the $\Delta \alpha, \Delta \delta$, treated as functions of the plate constants and the positions. Although parts of the AGK3 material have been sent to Lacroute by Dieckvoss in 1965, no positions according to the overlap method are available at present, so that there is no possibility of making a comparison with the classical reductions performed by Dieckvoss at the Hamburg Observatory. In general, the direct handling of spherical coordinates in overlap technique seems to be due to Eichhorn (1963).

Using the transformations (König, 1933) between the standard coordinates of two overlapping plates referred to different tangential points, de Vegt (1967) has recently set up a rigorous non-linear system of equations of condition involving as unknowns only the plate constants of all overlapping plates. Since the overlap condition is directly expressed in the equations of condition, no previous transformation to a common tangential point is necessary.

To have a comparison between the classical approach - involving only the reference stars - and the overlap solution, an artificial star field, generated by random numbers and divided into $132^{\circ} \times 2^{\circ}$ 'plates', was used to obtain 'true' positions, not affected by any error. These true positions were superimposed by different Gaussian distributions $N\left(0, \sigma_{i}\right)$ to get 'observed' positions on each plate. From the resulting field-star positions of both, the classical and the overlap reduction, one can compare (1) the internal scatter (m.e.) of the field-star positions (from the contributions of the neighbouring plates), and (2) the mean differences in the sense 'true minus computed position'. It was found that for the overlap solution the mean error of the positions is reduced by $10-15 \%$, and a better approximation of the true positions is obtained, that means: the overlap solution gives a better systematic representation of the desired position in comparison with the classical solution.

Furthermore, Clube (1967) has described a method to obtain accurate relative star positions from overlapping photographic plates. The configuration of stars in common to each pair of overlapping plates are adjusted for scale and orientation, so that the relative displacements on the celestial sphere of the stars in common are minimized. A direct non-linear relation between the plate constants and the equatorial direction cosines $\tau_{1}, \tau_{2}, \tau_{3}$ of the stars is used. Starting with an adopted set of provisional equatorial coordinates, the plate constants are computed to get improved direction cosines. Weighted means of the $\tau_{j}$ are then found from overlapping plates on which 
each star appears, and these are used as improved estimates in repeating the above procedure, so that an iterative process is set up. By this process, the overlapping plates should get a 'rigid connection' and only a few reference stars or starlike extragalactic objects should be necessary to transform the relative star positions to an inertial reference frame. The question of the convergence of the iterative process is under further investigation. Since the whole program is in the development stage, no practical results are available at present. Recently, Lukac (1967) used Eichhorn's overlap method in an investigation concerning relative star positions in the Pleiades cluster.

Some few remarks may be of interest concerning the mathematical problems arising from overlap solutions. In all cases, the resulting linear system of normal equations involves a large number of unknowns, especially if complete catalogue-zones are reduced as a whole, so that an iterative solution scheme would always be necessary. Since the matrix of the normal system is positive-definite some, a priori, convergent iterative methods are available, as the Gauss-Seidel method (Faddeev and Faddeeva, 1964) or gradient-methods. Of special advantage seems to be the method of groupiteration (Zadunaisky, 1957; Jefferys, 1963) because, on account of the special structure of the normal equations in most cases, a splitting up into block-type matrices is possible. A difficulty may further arise from the non-linearity of the equations of condition because the necessary neglecting of higher-order terms in the Taylor approximation may invalidate the linearisation process if the initially adopted approximate values for the unknowns differ too much from the exact values. (In the case of de Vegt's overlap solution it was found that the plate constants performed from the classical reduction gave a fairly good approximation.) If this situation prevails, the 'method of damped least squares' by Levenberg (1944) may give an improvement. A detailed discussion of the topological structure of the matrices resulting from overlap conditions is given by Henricksen (1967), who also gives an estimation of the needed machine hours for the reductions. It is shown that an iterative solution-procedure requires fewer operations than does a direct method in this kind of astrometric problems.

As is shown from the practical results of Eichhorn and de Vegt, an overlap solution seems to give a real improvement compared with the classical treatment. However, the question concerning a possible reduction of the numbers of reference stars in overlap techniques should be a matter of further discussion, although Eichhorn's result seems to give a positive hint. Recent investigations by Dieckvoss with regard to the AGK3 reductions have shown that the overlap method may fail if only a small number of common stars is available. Depending also on the accuracy of the measured $x, y$, the plates then cannot be so 'rigidly' connected by the overlap that the reduced number of reference stars will provide an accurate transformation to an inertial reference system. 


\section{References}

Clube, S.V.M. (1967) Private Communication.

de Vegt, Chr. (1967) Astr. Nachr., 290 (in press).

Eichhorn, H. (1960) Astr. Nachr., 285, 233.

Eichhorn, H. (1963) Private Communication.

Eichhorn, H., Googe, W.D., Gatewood, G. (1967a) Astr. J., 72, 626.

Eichhorn, H., Gatewood, G.D. (1967b) Astr. J. (in press).

Faddeev, D. K., Faddeeva, V.N. (1964) Numerische Methoden der linearen Algebra.

Googe, W.D. (1967) Astr. J., 72, 623.

Henricksen, S. W. (1967) Astr. J., 72, 603.

Jefferys, H. III (1963) Astr. J., 68, 111.

König, A. (1933) Handbuch der Astrophysik, 1, 508ff.

Lacroute, P. (1964) Ann. Obs. Strasbourg, 6.

Levenberg, K. (1944) Quart. of Appl. Math., 2.

Lukac, C.F. (1967) Astr. J., 72, 620.

Zadunaisky, P. (1957) I.B.M. Watson Lab. Report. 